\title{
Apela/Elabela/Toddler: New perspectives in molecular mechanism of heart failure
}

Department of Biochemistry and Molecular Biology, Faculty of Medicine, Tarumanagara University, Jl. Letjen S. Parman No. 1, Jakarta, Indonesia

*Email: angelafelicia.s@gmail.com
Anthony P. Sunjaya, Angela F. Sunjaya*, Frans Ferdinal

\section{ABSTRACT}

Background. Despite significant therapeutic advances, heart failure (HF) remains unacceptably high in morbidity and mortality. Additionally, its high-care and costs make HF a deadly and costly disease. First reported independently by two group of researchers, Apela/Elabela/Toddler (ELA) is the second endogenous apelin-receptor ligand discovered which is encoded from a previously classified non-coding gene, and has emerged as a key signalling-pathway in the cardiovascular system.

Aims. To explore and summarise the biological effects and diagnostic potential of ELA as a new biomarker for heart failure.

Results. ELA (prepro-ELA $54 \mathrm{AA}$ ) is a molecule with three isoforms (ELA 11,16 and 32), recently identified as the second endogenous ligand to APJ-receptor and functions to mediate early cardiac development during zebrafish embryogenesis by inducing cardiogenesis, vasculogenesis and bone formation. In adults, it enhances cardiac contractility, promotes vasodilatory effects, mediates fluid homeostasis, reduces food intake, limits kidney dysfunction and exerts antiatherosclerotic as well as anti-oxidative properties.

Conclusion. These results show that ELA, an endogenous agonist of the APJ-receptor exerts cardiovascular effects comparable and potentially more potent than apelin and is found to be downregulated in experimental models and humans with heart failure. https://doi.org/

10.21542/gcsp.2019.15

Received: 08 May 2019 Accepted: 18 June 2019 (C) 2019 The Author(s), licensee Magdi Yacoub Institute. This is an open access article distributed under the terms of the Creative Commons Attribution license CC BY-4.0, which permits unrestricted use, distribution and reproduction in any medium, provided the original work is properly cited.
Cite this article as: Sunjaya AP, Sunjaya AF, Ferdinal F. Apela/Elabela/Toddler: New perspectives in molecular mechanism of heart failure, Global Cardiology Science and Practice 2019:15

https://doi.org/10.21542/gcsp.2019.15 


\section{INTRODUCTION}

Heart failure is a complex clinical syndrome resulting in a reduced ability of the heart to pump and/or fill with blood, and is considered the fatal finishing line of all cardiovascular disorders. Despite significant therapeutic advances, heart failure (HF) remains unacceptably high in morbidity and mortality. Additionally, its high care requirements and associated costs make HF not only a deadly disease, but also a costly one. In 2014 an estimated 26 million people worldwide suffered with heart failure. In 2012 the United States reported over 10\% of its total health expenditure (USD 31 billion) was spent on cardiovascular diseases with total costs expected to increase by $127 \%$ between 2012 and 2030 ${ }^{1,2}$.

Significant efforts have been made in searching for new HF biomarkers ${ }^{3}$. First reported independently by two different group of researchers - Chng et al. ${ }^{4}$ and Pauli et al ${ }^{5}$, Apela/Elabela/Toddler (ELA) is the second endogenous apelin-receptor ligand discovered encoded from a previously classified non-coding gene and has emerged as a key signalling-pathway in the cardiovascular system.

\section{Apelin-APJ axis}

Apelin is an endogenous hormone that binds to apelin receptors and exerts potent positive inotropic activity and increases coronary blood flow by vascular dilation, thereby providing beneficial effects in failing hearts ${ }^{6}$. The APJ, or apelin, receptor is a G-protein coupled receptor which shares similarity in homology to that of the angiotensin II type 1 receptor (54\% homology in transmembrane domains and 32\% homology for the entire sequence $)^{7,8}$. Hence, the APJ system was found to negatively regulate angiotensin II signalling and upregulates angiotensin-converting enzyme 2 (ACE2) expression ${ }^{9}$. The Apelin-APJ axis was reported from mouse models to maintain heart contractility and cardioprotective effects under conditions of pressure overload, exercise and ageing ${ }^{10,11}$.

\section{Elabela/Toddler/Apela (ELA)}

Earlier apelin has been thought to be the only ligand of the apela receptor, however recently ELA has been identified as the second endogenous ligand to this receptor. ELA (prepro-ELA $54 \mathrm{AA}$ ) is a molecule with 3 isoforms (ELA 11,16 and 32 ) $^{12}$ and functions to mediate endoderm differentiation and cell migration hence promoting early cardiac development during zebrafish embryogenesis by inducing cardiogenesis, vasculogenesis and bone formation. Severe developmental defects including rudimentary or absent heart formations have been reported following ELA mutation similar to the effects of mutation of the Apela receptor 4,5 (Figures 1 and 2).

Recent studies have also shown the potential effects of ELA in the self-renewal of human embryonic stem cells (hESC) in an autocrine manner which may be a result of its interaction with another unknown alternate receptor (receptor X) ${ }^{13}$ (Figure 2).

In adults, ELA has been found localized in the endothelium and has an affinity for the human apelin receptor ${ }^{4}$. In addition, ELA has also been reported to be detected only in human stem cells (embryonic and induced adult pluripotent stem cells), and kidney unlike APJ receptors which have been reported to be found in heart, kidney, brain, lung, stomach, pancreas, fats and stem cells ${ }^{13,15}$. Hence, ELA was found to enhance cardiac contractility, promote vasodilatory effects, mediate fluid homeostasis, reduce food intake, limit kidney dysfunction and exerts anti-atherosclerotic as well as anti-oxidative properties $^{16}$ (Figure 3).

With regards to fluid homeostasis, due to its greater distribution in the kidneys than apelin, ELA infusion resulted in a response 5 times that of apelin ${ }^{17}$. ELA treatment 
A ELABELA
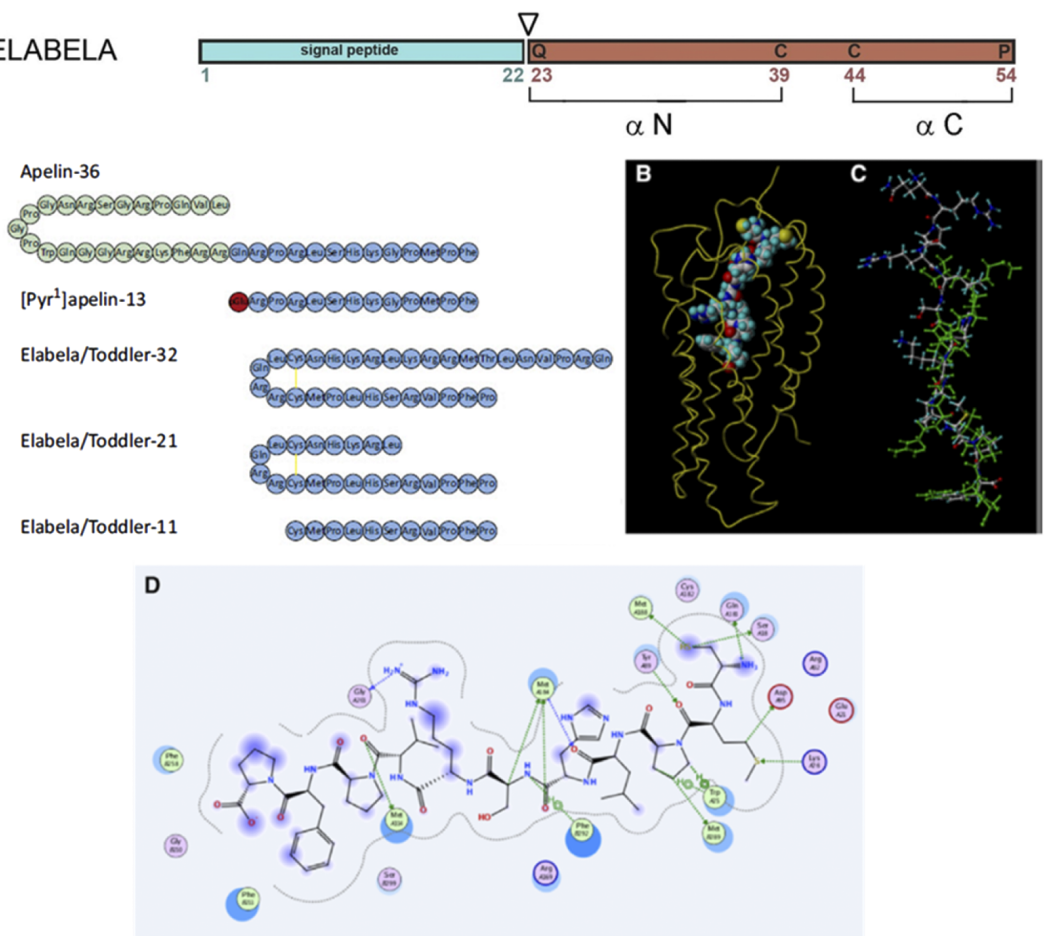

Figure 1. A - Sequence of apelin and Elabela/Toddler peptides ${ }^{4,14}$; B - Docking of ELA-11 on the apelin receptor; $\mathrm{C}$ - Apelin-13 (multicolored atoms) and ELA-11 (green) docking showing overlap in the binding site; D - Ligand interaction diagram showing key close contacts between ELA-11 and the apelin receptor model; ELA indicates Elabela/ Toddler/Apela ${ }^{12}$.

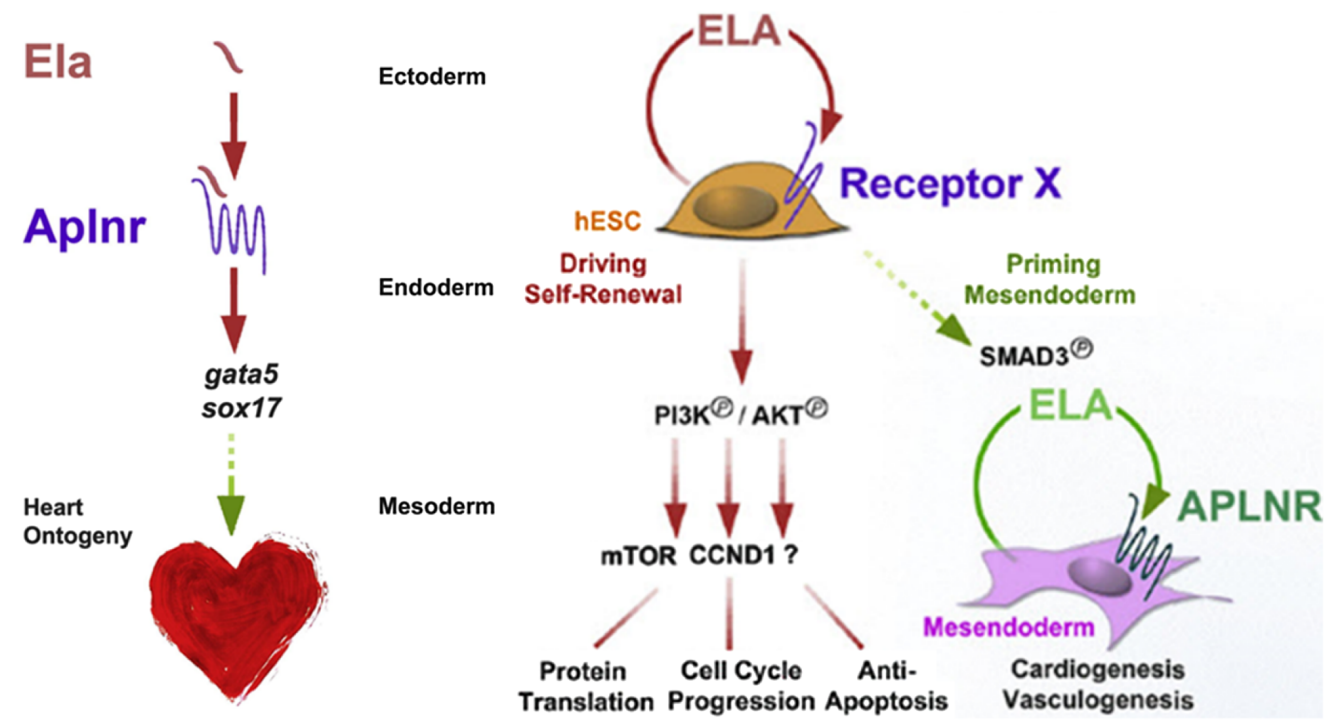

Figure 2. ELA's effect during cardiogenesis ${ }^{4,13}$.

significantly suppressed the increased expression of mRNA associated with cardiac hypertrophy, such as brain natriuretic peptide (BNP), atrial natriuretic factor (ANF), and b-myosin heavy chain (b-Myhc) ${ }^{7}$, as well as markers of markers of myocardial injury such as lactate dehydrogenase (LDH), creatinine kinase-MB (CK-MB), Troponin I and markers of oxidative stress such as membrane lipid peroxidation (MDA), glutathione (GSH) and 


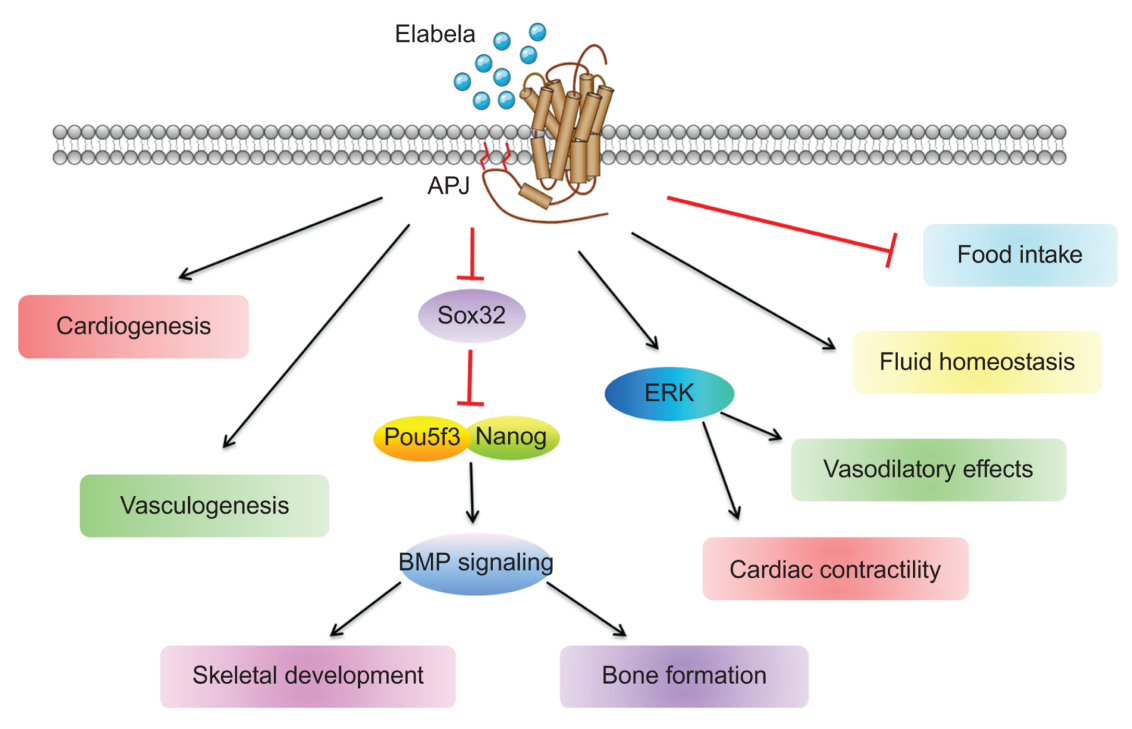

Figure 3. ELA's effect during embryogenesis and in adults ${ }^{16}$.

superoxide dismutase activities (SOD). Animal studies have also reported that cardiac function as measured by echocardiography improved significantly 2 weeks after ELA infusion post myocardial infarction ${ }^{18}$.

\section{CONCLUSION}

These results show that ELA is an endogenous agonist of the APJ receptor exerting cardiovascular effects comparable and potentially more potent than apelin and downregulated in experimental models and humans with heart failure. ELA's discovery open new possibilities in the field of biomarkers and therapeutics of heart failure due to its significance in the pathophysiology of heart failure. Further studies on ELA and APJ signalling are needed to provide more mechanistic insights into regulation and activation of APJ in cardiovascular diseases.

\section{CONFLICT OF INTEREST}

The authors declare no conflict of interest with regards to this publication.

\section{REFERENCES}

[1] Iqbal N, Wentworth B, Choudhary R, Landa AD, Kipper B, Fard A, et al. Cardiac biomarkers: new tools for heart failure management. Cardiovasc Diagn Ther. 2012;2(2).

[2] Savarese G, Lund LH. Global Public Health Burden of Heart Failure. Card Fail Rev. 2017;3(1).

[3] Correale M, Monaco I, Brunetti ND, Biase MD, Metra M, Nodari S, et al. Redefining biomarkers in heart failure. Heart Failure Reviews. 2018.

[4] Chng SC, Ho L, Tian J, Reversade B. ELABELA: a hormone essential for heart development signals via the apelin receptor. Developmental Cell. 2013;27(6).

[5] Pauli A, Norris ML, Valen E, Chew GL, Gagnon JA, Zimmerman S, et al. Toddler: an embryonic signal that promotes cell movement via Apelin receptors. Science. 2014;343(6172).

[6] Japp AG, Cruden NL, Barnes G, van Gemeren N, Mathews J, Adamson J, et al. Acute cardiovascular effects of apelin in humans: potential role in patients with chronic heart failure. Circulation. 2010;121.

[7] Sato T, Sato C, Kadowaki A, Watanabe H, Ho L, Ishida J, et al. ELABELA-APJ axis protects from pressure overload heart failure and angiotensin II-induced cardiac damage. Cardiovascular Research. 2017;113.

[8] Xu J, Chen LX, Jiang Z, Li L. Biological functions of Elabela, a novel endogenous ligand of APJ receptor. Journal of Cellular Physiology. 2018.

[9] Sato T, Suzuki T, Watanabe H, Kadowaki A, Fukamizu A, Liu PP, et al. Apelin is a positive regulator of ACE2 in failing hearts. J Clin Invest. 2013;123. 
[10] Kuba K, Zhang L, Imai Y, Arab S, Chen M, Maekawa Y, et al. Impaired heart contractility in Apelin genedeficient mice associated with aging and pressure overload. Circ Res. 2007;101.

[11] Charo DN, Ho M, Fajardo G, Kawana M, Kundu RK, Sheikh AY, et al. Endogenous regulation of cardiovascular function by apelin-APJ. Am J Physiol Heart Circ Physiol. 2009;297.

[12] Yang P, Read C, Kuc RE, Buonincontri G, Southwood M, Torella R, et al. Elabela/Toddler is an endogenous agonist of the apelin APJ receptor in the adult cardiovascular system, and exogenous administration of the peptide compensates for the downregulation of its expression in pulmonary arterial hypertension. Circulation. 2017;135.

[13] Ho L, Tan SY, Wee S, Wu Y, Tan SJ, Ramakrishna NB, et al. ELABELA is an endogenous growth factor that sustains hESC self-renewal via the Pl3K/AKT pathway. Cell Stem Cell. 2015;17.

[14] Yang P, Maguire JJ, Davenport AP. Apelin, Elabela/Toddler, and biased agonists as novel therapeutic agents in the cardiovascular system. Trends Pharmacol Sci. 2015;36(9).

[15] Wang Z, Yu D, Wang M, Wang Q, Kousnetsova J, Yang R, et al. Elabela-apelin receptor signaling pathway is functional in mammalian systems. Sci Rep. 2015;5.

[16] Lu L, Cao J, Li L, Chen L. Elabela, a new endogenous ligand of APJ, functions in embryos and adults organisms. Acta Biochimica et Biophysica Sinica. 2017;49(4).

[17] Deng C, Chen H, Yang N, Feng Y, Hsueh AJ. Apela regulates fluid homeostasis by binding to the APJ receptor to activate GI signalling. Journal of Biological Chemistry. 2015;290(30).

[18] Rakhshan K, Azizi Y, Naderi N, Afousi AG, Aboutaleb N. ELABELA (ELA) peptide exerts cardioprotection against myocardial infarction by targeting oxidative stress and the improvement of heart function. International Journal of Peptide Research and Therapeutics. 2018. 